Annuaire suisse de politique de développement

2 | 1982

Annuaire Suisse - Tiers Monde 1982

\title{
3. Coopération au développement
}

\section{Hilmar Stetter}

\section{OpenEdition}

\section{Journals}

Édition électronique

URL : http://journals.openedition.org/aspd/1120

DOI : 10.4000 /aspd. 1120

ISSN : 1663-9669

\section{Éditeur}

Institut de hautes études internationales et du développement

\section{Édition imprimée}

Date de publication : 31 août 1982

Pagination : 81-101

ISSN : 1660-5934

\section{Référence électronique}

Hilmar Stetter, «3. Coopération au développement », Annuaire suisse de politique de développement [En ligne], 2 | 1982, mis en ligne le 20 janvier 2013, consulté le 08 septembre 2020. URL : http:// journals.openedition.org/aspd/1120; DOI : https://doi.org/10.4000/aspd.1120 


\section{COOPERATION AU DEVELOPPEMENT}

En 1981, la coopération au développement s'est ressentie en Suisse des coupes linéaires de $10 \%$ pratiquées dans le budget de la Confédération. Le Parlement a décidé de lui retirer encore une nouvelle tranche de 8\% pour 1982. Même si le volume des fonds dont elle dispose est en légère augmentation par rapport au PNB, elle piétine et demeure en dessous de la movenne internationale. En décembre 1981, le Parlement a voté un nouveau crédit-cadre de 360 millions de francs destiné à financer des mesures d'aide humanitaire internationale. Enfin, le Conseil fédéral a souscrit à l'accord passé entre la Confédération et "Intercoopération".

\section{LES DEPENSES DE LA COOPERATION AU DEVELOPPEMENT EN 1981}

En 1981, la Suisse a consacré à la coopération au développement (Confédération, cantons et communes) 452,6 millions de francs, ce qui représente $0,24 \%$ du PNB $, 0,01 \%$ de plus qu'en 1980. Le budget de 1982 porte sur un montant de 481,2 millions de francs, soit une augmentation de $5,8 \%$. On en resterait à $0,24 \%$ du $\mathrm{PNB}(1)$.

Mesurées à l'échelle du PNB, les dépenses de la Suisse en fonds publics pour la coopération au développement restent stagnantes et sont en dessous de la moyenne enregistrée dans les pays industriels. Par tête d'habitants, la Suisse ne contribue que bien peu à I'aide publique au développement : 71 francs. Elle est loin d'atteindre l'objectif de 0,7\% du PNB pour la coopération au développement défini dans la stratégie de l'ONU pour le développement au cours des années 80 , stratégie à laquelle la Suisse a souscrit au demeurant. La part de la Suisse à I'aide publique au développement des pays du CAD s'est élevée en 1981 à $0,93 \%$ du total.

La coopération au développement assumée par la Confédération est financée 
à partir de divers crédits-cadres, gérés par plusieurs départements, à savoir :

- le crédit-cadre de 1.650 millions de francs du 8 décembre 1980, pour la coopération technique et l'aide financière;

- le crédit-cadre de 270 millions de francs du 14 mars 1979 pour l'aide humanitaire internationale. Ce crédit est échu le 31 mars 1982. Un nouveau crédit de 360 millions de francs a été accordé le 3 décembre 1981 et est entré en vigueur en avril 1982;

- le crédit-cadre de 300 millions de francs du 26 septembre 1979 pour les augmentations de capital des banques interaméricaine, africaine et asiatique de développement;

- le crédit-cadre de 200 millions de francs du 28 novembre 1978 pour le financement des mesures d'ordre économique et commercial prises dans l'optique de la coopération au développement. Ce crédit avait été entièrement utilisé au milieu de l'année; un nouveau crédit de 350 millions de francs a été proposé au Parlement;

- le crédit-cadre de 9,2 millions de francs du 19 décembre 1980 pour l'octroi de bourses à des étudiants étrangers en Suisse. La majeure partie de ce crédit est attribuée à des étudiants en provenance de pays du Tiers Monde (2).

En définissant les directives de sa politique pour la législature 1979-83, le Conseil fédéral s'était promis d'augmenter fortement ses dépenses en faveur de la coopération au développement. II entendait se rapprocher du niveau moyen des prestations des pays du CAD, qui s'établit à environ $0,35 \%$ de leur PNB. Au cours des années à venir, il désire atteindre cette moyenne. Exprimées en chiffres, les dépenses totales pour la coopération publique au développement figurent ainsi au plan financier de la législature en cours :

\begin{tabular}{lcc} 
Année & Montant (millions de fr.) & Part du PNB \\
\hline 1981 & 487,8 & $0,27 \%$ \\
1982 & 598,8 & $0,31 \%$ \\
1983 & 618,8 & $0,31 \%$
\end{tabular}

Selon les mesures d'économie décrétées en 1980 par la Confédération, le plan des investissements financiers pour l'aide au développement prévoit des réductions annuelles de $10 \%$. Les prestations de la Confédération à elle seule (sans les cantons et les communes) ont encore atteint le niveau de 446,5 millions de francs, c'est-à-dire $2,6 \%$ de l'ensemble du budget ou $0,24 \%$ du PNB.L'augmentation par rapport à 1980 a été en valeur nominale de $10 \%$, surtout à cause de l'octroi d'un crédit d'aide économique à la Turquie (3).

\section{Répartition des crédits}

En 1981, la part des crédits pour la coopération technique n'a guère varié (augmentation de $2 \%$ ). II en a été de même pour l'aide alimentaire et huma- 
nitaire (en recul toutes les deux d'environ 2,8\%). Portées au compte de l'aide financière, les mesures d'aide économique et commerciale ont progressé (de $4 \%$ à $14 \%$ ), alors que l'aide financière représentait $21 \%$ du total (contre $30 \%$ l'année précédente) (4).

Dans l'ensemble des prestations, la part des dons s'est élevée à $88,8 \%$, ce qui représente une diminution par rapport à $1980(97,1 \%)(5)$.

La part de l'aide bilatérale s'est à peine modifiée $(64,5 \%$ contre $64,8 \%$ l'année précédente). Par contre, la ventilation géographique a évolué. La part de I'Afrique a connu une nouvelle et légère progression, passant à $42,4 \%$, alors que I'Amérique latine est un peu en recul, l'Asie et l'Océanie en forte régression, passant de $36,6 \%$ à $26,9 \%$. Etonnante progression de l'Europe, dont la part s'élève de $0,2 \%$ à $7,5 \%$, ce qui est dû à l'octroi du crédit d'aide économique à la Turquie déjà mentionné (6).

Si l'on répartit l'aide bilatérale selon le revenu des pays bénéficiaires, on verra qu'en 1981, ce sont de nouveau des pays les moins avancés qui en ont reçu la plus grande part, soit 39\%. La part des pays en développement jouissant d'un revenu moyen a passé à $22 \%$ contre $16 \%$ en 1980 , encore une conséquence de l'aide apportée à la Turquie. Les autres pays à faible revenu ont reçu $18 \%$ de I'aide bilatérale (contre $25 \%$ en 1980) (7).

\section{Les tournants de la politique de développement}

Cette répartition quantitative ne dit pas tout sur les activités de la coopération suisse au développement et sur les décisions importantes prises au cours des douze derniers mois. Le débat sur la coopération suisse au développement qui avait été amorcé au début de 1981 s'est poursuivi dans les commissions, au Parlement et dans les médias. On s'est moins attaché à la discussion des principes et de la nécessité de la coopération suisse au développement, mais plutôt à certains instruments qu'elle utilise et à certaines des mesures prises. On a mis I'accent sur ses relations avec l'économie privée, sur la transparence de son action, sur la politique d'information des instances fédérales chargées de la réalisation de ses objectifs.

En ce qui concerne les montants attribués à l'aide au développement, les deux Chambres ont accepté les coupes linéaires de $10 \%$ du budget et la réduction additionnelle de $8 \%$. La proposition Bauer-Lagier (PL, GE) demandant que la coopération au développement soit totalement exonérée de cette mesure a été repoussée au Conseil des Etats par 21 voix contre 8 . Au Conseil national la proposition Ott (PS, BL) concernant l'exemption de la coopération technique de la réduction de $8 \%$ fut repoussée par 76 voix contre 59. La commission des finances et le Conseil fédéral s'étaient prononcés contre cette proposition. La communauté de travail Swissaid/Pain pour le prochain/Action de Carême/Helvetas s'était en revanche élevée contre ces réductions budgétaires "à la charge des pauvres" (8).

Le Parlement eut enfin à se prononcer sur deux nouveaux crédits-cadres : 
- 350 millions de francs pour le financement des mesures d'ordre économique et commercial à prendre dans l'optique de la coopération au développement. Ce crédit-cadre remplace le crédit de $\mathbf{2 0 0}$ millions de francs qui arrive à échéance et couvre des mesures à prendre dans le domaine des matières premières, du commerce, de l'industrialisation et des finances (crédits mixtes et aide à l'équilibre de la balance des paiements). Les prestations qui en découlent s'étendront sur une période de cinq à sept ans. Le crédit a été accepté par le Conseil national à la date du 23 juin 1982. II sera présenté au Conseil des Etats lors de la session d'automne.

- 360 millions de francs pour l'aide humanitaire. Ce crédit-cadre finance également des mesures à prendre au cours des trois années à venir, au minimum dès le 1er avril 1982, date à laquelle le dernier crédit de $\mathbf{2 7 0}$ millions de francs est arrivé à échéance.

En décembre 1981, le Conseil fédéral a accepté un accord intervenu entre la Confédération et la nouvelle organisation "Intercoopération" qui regroupe sept œuvres d'entraide suisses. "Cette collaboration fait partie des mesures permettant à la Confédération de maintenir à un niveau constant la qualité de la coopération suisse au développement dans son expression bilatérale en tenant compte de l'augmentation des engagements et de l'effectif plus ou moins stable du personnel" (9).

La conclusion de cet accord avait été précédée par une discussion soutenue au Parlement et dans la presse. Des firmes d'ingénierie et d'autres représentants de l'économie ont regretté à ce propos les contacts insuffisants, selon leurs vues, de la DDA avec l'économie. Pour sa part, l'administration a pu faire la preuve des nombreux contacts qu'elle avait établis et, pour la première fois, elle a publié des chiffres sur les bénéfices encaissés par l'économie privée et provenant de la coopération au développement. Afin d'améliorer l'information à l'intention de l'économie privée, la DDA a mis sur pied un programme d'information sur les projets et les programmes futurs de la coopération technique et de l'aide financière (10).

La commission consultative pour la coopération internationale au développement s'est également occupée de manière soutenue de la politique suisse de développement. On y a discuté entre autres, avec la commission de consultation pour la politique commerciale, du renouvellement du crédit-cadre pour les mesures d'ordre économique et commercial, du processus de décision pour les projets de la coopération technique, de l'aide financière et de l'octroi de crédits mixtes. Lors de sa dernière session du premier semestre de 1982, la commission se pencha sur le budget 1983 de la coopération au développement et s'occupa de l'état des évaluations de projets (11).

Le 17 septembre 1981, la DDA a pu se prévaloir, lors de sa conférence annuelle, de vingt ans d'activité. Elle y trouva I'occasion pour comparer son action et ses programmes à ceux de cinq pays hautement industrialisés (Danemark, Italie, Canada, Pays-Bas, Suède). Elle fit également face aux critiques dirigées contre elle. Le Conseiller fédéral Aubert ne manqua pas de 
souligner que la solidarité avec les pauvres et l'aide aux pays en développement sont fondamentalement nécessaires et utiles. Cette conférence annuelle servit de prétexte à la parution de diverses publications (12).

II y a eu enfin un important changement à la tête du personnel de la DDA. Son directeur, I'ambassadeur Marcel Heimo, a pris sa retraite à fin 1982. Pour lui succéder, le Conseil fédéral a désigné Fritz Staehelin en juin 1982. De 1970 à 1975, Fritz Staehelin a dirigé le service de développement de I'actuel OFAEE; il était depuis 1980 ambassadeur à Tokyo (13).

\section{Comparaison de l'aide publique et de l'aide privée au développement}

Si les apports de la Suisse à l'aide publique au développement restent en dessous de la moyenne, les prestations financières de source privée sont importantes dans les pays en développement. La Suisse figure au rang des grands fournisseurs de capitaux pour les pays en développement, avec la Belgique, la France, la RFA, I'Italie, le Japon, la Grande-Bretagne et les Etats-Unis. Selon le PNB par habitant, la Suisse est depuis plusieurs années et de loin en tête de liste des pays du CAD pour ses apports en aide privée au développement. En 1981, le flux net des capitaux privés suisses vers les pays en développement a été environ neuf fois plus élevé que les prestations publiques pour l'aide au développement, soit $3.888,5$ millions de francs. Ce qui équivaut pour 1981 à une propostion de 2,03\% du PNB. Pour les pays du CAD, I'aide privée moyenne enregistrée en 1981 s'établissait à près de $0,74 \%$ du PNB (14).

Quant à la part de l'aide publique au développement des dix-sept pays membres du CAD, elle représentait en $198131 \%$ de l'ensemble de leurs flux financiers en direction des pays en développement. Pour la Suisse, la proportion n'était que de $10,1 \%$ (15).

\section{MESURES ECONOMIQUES ET COMMERCIALES}

Le crédit-cadre arrivant à échéance doit être remplacé par un nouveau crédit de 350 millions de francs. Lors de la discussion devant le Conseil national, les mesures prévues ont été approuvées (matières premières, expansion commerciale, industrialisation, aide à l'équilibre des balances des paiements et crédits mixtes). Le nouveau crédit-cadre fait que ces diverses mesures figurent désormais pour une proportion de $15 \%$ au total de l'aide publique au développement.

C'est sans aucune opposition que le Conseil national a approuvé le 23 juin 1982 un crédit-cadre de 350 millions de francs qui doit servir à financer des 
mesures d'ordre économique et commercial dans l'optique de la coopération au développement. Le Conseil des Etats se prononce au cours de sa session de septembre.

Lors de la discussion du crédit au Conseil national et au sein de la commission compétente, on a remis en question les crédits mixtes, l'une des mesures prévues. On s'est interrogé sur leur utilité dans l'optique du développement et on a relevé que les crédits mixtes accordés à ce jour n'avaient guère été soumis à évaluation. On a affirmé que ces crédits mixtes en augmentation créaient un dilemme accru entre développement et politique commerciale. Liés à la fourniture de produits suisses, ils sont une source de conflits entre les intérêts de l'industrie d'exportation et les impératifs du développement dans les pays de destination. L'intérêt égoöste peut prévaloir, c'est là le danger (16). Au cours du débat, le Conseil fédéral fit remarquer que l'évaluation des crédits mixtes arrivés à échéance était en cours et que les premiers rapports seraient sans doute disponibles en 1983 (17).

La communauté de travail Swissaid/Pain pour le prochain/Action de Carême/ Helvetas proposa de maintenir à $10 \%$ la part attribuée aux mesures d'ordre économique et commercial et de ne pas porter cette proportion à $15 \%$ de l'aide publique au développement. Aussi longtemps qu'on ne dispose pas d'évaluations des crédits mixtes permettant des conclusions positives dans l'optique du développement, on ne devrait en augmenter les montants qu'en proportion des crédits globaux. On pourrait ainsi réduire le crédit-cadre à 300 millions de francs ou en prolonger la durée de six mois (18). Les parlementaires $n$ 'ont cependant pas tenu compte de ces considérations.

Le crédit-cadre de 350 millions de francs aurait dû à l'origine entrer en vigueur en 1er juillet 1982 et avoir une durée minimum de trois ans. Les versements s'étendent sur un laps de cinq à sept ans. Ce crédit prend le relais du crédit de 200 millions de francs qui était entré en vigueur en 1978 et qui avait été entièrement utilisé à mi-1982.

Cependant, l'octroi du crédit-cadre ne signifie pas qu'il doive être dépensé au cours des trois années qui viennent. II se borne à mettre le Conseil fédéral en mesure de faire face aux obligations découlant des diverses mesures prises et à effectuer les paiements qui s'imposent. Certaines mesures s'étendent sur plusieurs années; le volume global des prestations doit être évalué au stade des préparatifs. Le montant du crédit est déterminé par l'ensemble des dépenses pour l'aide au développement portées au plan financier, la part des divers crédits-cadres et la durée des engagements.

Au cours de la présente législature, les dépenses suivantes ont été engagées ou portées au budget pour des mesures d'ordre économique et commercial (en \% du budget global de la coopération au développement) :

$\begin{array}{lllll}1979 & 1980 & 1981 & 1982 & 1983 \\ 5,2 \% & 9,6 \% & 10,8 \% & 10,4 \% & 13,3 \%\end{array}$


Si l'on tient compte des engagements découlant des autres crédits-cadres en cours, la part prise par les mesures économiques et commerciales s'accroît ainsi d'année en année. Selon le message à moyen terme, la part de ces mesures doit s'établir à environ $15 \%$. La commission consultative pour la politique commerciale et la commission d'experts de la coopération internationale au développement ont donné leur accord. La part proportionnelle de la coopération technique et de l'aide financière se trouve réduite d'autant (19).

Le crédit échu de 200 millions de francs

Le crédit arrivant à échéance avait été engagé à près de $80 \%$ à fin septembre 1981, son utilisation est la suivante :

\begin{tabular}{ccc}
$\begin{array}{c}\text { Montant } \\
\text { prévu }\end{array}$ & $\begin{array}{c}\text { Dépenses } \\
\text { engagées }\end{array}$ & Reliquat \\
\hline
\end{tabular}

en millions de francs suisses

\begin{tabular}{lrrr}
\hline & & & \\
Crédits mixtes & 110 & 102,50 & 7,50 \\
Aide à la balance des paiements & 50 & 33,00 & 17,00 \\
Matières premières & 25 & 20,70 & 4,30 \\
Expansion commerciale en faveur & 10 & 1,20 & 8,80 \\
des pays en développement & 5 & 2,70 & 2,30 \\
Industrialisation & 200 & 160,10 & 39,90 \\
\cline { 2 - 4 } TOTAL & & & \\
\hline
\end{tabular}

Source : BBL no 12, 1982, p. 726.

Ces deux dernières années, les crédits-cadres ont été utilisés (1981) ou portés au budget (1982) ainsi (20) :

1981 (Fr.)

Matières premières

Expansion commerciale

Industrialisation

Aide à la balance des

paiements

Crédits mixtes

Mise en œuvre
1982 (Fr.)

100.000

11.500 .000

2.000 .000

2.000 .000

694.663

9.000 .000

19.000 .000

124.000 
Les contributions suisses à l'accord international pour les produits de base sont financées par des mesures prises dans le domaine des matières premières; il en va de même pour les projets destinés à améliorer la production, le transport et la commercialisation des matières premières. Le poste le plus important sera la contribution de la Suisse au Fonds commun pour les produits de base, qui n'a pas encore été versée. Le même fonds fournira les contributions aux accords internationaux sur le caoutchouc naturel et l'étain.

Dans le cadre de la promotion commerciale, la mesure financière la plus importante est la participation de la Confédération à la présence (préparatifs et réalisation) de trois pays en développement à des foires suisses :

l'Egypte, le Sénégal et le Sri Lanka. Ont également été financés entièrement ou en partie un cours de politique commerciale organisé par le GATT à l'intention de spécialistes de l'exportation latino-américaine, des projets du $\mathrm{CIC}$ (Centre international du commerce CNUCED/GATT Genève) et d'OS3 (Organisation Suisse-Tiers Monde, Sonceboz) afin de lui permettre d'importer davantage de produits en jute à l'échelle internationale, et notamment de stimuler le marché suisse.

Dans le domaine de l'industrialisation, on désire encourager par des transferts de technologie et des investissements privés suisses les efforts des pays en développement. C'est le bureau de I'ONUDI à Zurich qui joue le rôle d'intermédiaire. II dispose d'un fonds spécial pour les études de pré-investissement des projets industriels. Trois projets d'investissements industriels au Sri Lanka ont à ce jour bénéficié de ce soutien (fabrication d'emballages pour les exportations de thé, production de boîtes à musique pour l'exportation, sucreries).

L'aide à la balance des paiements a pour but de garantir la capacité d'importation de divers pays en développement dans le cadre de mesures coordonnées à l'échelle internationale et de soulager ainsi leur potentiel de production. Elle contribue aussi à équilibrer la balance des paiements et à maintenir à un niveau normal le trafic international des paiements et des marchandises du pays en développement intéressé. Elle répond également à l'intérêt que la Suisse porte à un bon fonctionnement du système international des paiements et à une politique commerciale libérale.

Dans le cadre d'un crédit de programmes d'importation de I'AID se montant à 74 millions de dollars EU, le Bangla Desh a reçu une contribution non remboursable de 15 millions de francs (9 millions de dollars EU). Ce crédit permettra de mettre en œuvre un programme national de développement de la production vivrière au moyen d'importations de matières premières, de produits semi-finis et de pièces de rechange (21).

Aux neuf crédits mixtes accordés à fin Juin 1981, quatre autres sont venus s'ajouter à fin juin 1982 : pour le Zimbabwe (premier et deuxième accords), le Kenya, le Maroc et le Honduras. Le crédit accordé à l'Egypte est épuisé, les autres en cours d'utilisation. Diverses tranches d'autres crédits ont été versées à I'Inde, à la Tunisie, à la Thaïlande, au Sri Lanka et au Sénégal (22). 
Le "Comité suisse de soutien aux Sahraouis" a déposé le 10 août 1981, contre le crédit mixte accordé au Maroc, une pétition demandant l'interruption de la coopération au développement avec le Maroc aussi longtemps que ce pays participe à des opérations de guerre. Le Conseil fédéral a été prié de faire parvenir une aide humanitaire aux réfugiés saharaouis (23).

Le même crédit a été critiqué au Parlement au cours de diverses interventions personnelles, soit à cause du conflit en cours, soit du fait de la politique de développement du Maroc, orientée vers les exportations. Le Conseil fédéral estime que, malgré la guerre, le crédit influencera favorablement la politique économique marocaine jugée propice au développement (24).

\section{Le nouveau crédit-cadre}

Le nouveau crédit-cadre concerne essentiellement les cinq domaines suivants: matières premières, expansion commerciale des pays en développement, industrialisation, crédits mixtes et balance des paiements. Sa répartition est la suivante (25) :

Crédits mixtes et mesures analogues

Aide à la balance des paiements

Matières premières

Expansion commerciale

Industrialisation
240 millions

70 millions

20 millions

10 millions

10 millions

Divers objectifs du développement sont touchés par les mesures envisagées :

"Grâce à l'aide financière, crédits mixtes y compris, nous contribuons à l'amélioration de l'infrastructure sociale et économique. Les mesures économiques et commerciales ont pour but de mieux associer les pays en développement au commerce mondial afin qu'ils en retirent davantage de profit.

Elles servent aussi à calmer les perturbations des échanges internationaux et du trafic des paiements. II y a lieu enfin d'encourager les investissements de l'économie privée qui favorisent le développement au sens des dispositions de la loi sur la coopération au développement" (26).

Les pays bénéficiant des mesures évoquées plus haut auraient déjà quelque peu progressé sur la voie du développement. Ces mesures s'adressent plutôt aux pays pauvres qui ont un revenu un peu meilleur par tête d'habitant (exception possible : la balance des paiements). On ne peut souvent les appliquer qu'à l'ensemble des pays en développement ou à un groupe d'entre eux (action indirecte). Les liens avec l'économie suisse au sens étroit et les intérêts généraux de la politique étrangère du pays intéressé comptent davantage que les motivations humanitaires et politiques de la coopération au développement (27). 


\section{LES BANOUES REGIONALES DE DEVELOPPEMENT}

La Banque interaméricaine de développement (BID), dont la Suisse fait partie depuis 1976, s'est adressée en 1981 au marché suisse des capitaux, comme les années précédentes. Elle y a contracté des engagements portant sur 129,9 millions de dollars EU :

- emprunt 1981-91 à 7\% de 100 millions de francs d'un consortium placé sous la direction de trois grandes banques suisses;

- placement privé 1981-86 à $81 / 4 \%$ de 80 millions de francs d'un consortium de banques suisses dirigé par l'Union de banques suisses;

- emprunt 1981-91 à $81 / 2 \%$ de 80 millions de francs d'un consortium des trois grandes banques.

A fin 1981, les emprunts contractés par la BID s'élevaient ainsi à 3,476 milliards de dollars EU, dont 0,8 milliard en Suisse. Après les EU, la place financière suisse fut ainsi le principal fournisseur de crédits de la banque. La BID a contracté d'autres emprunts en Suisse durant le premier semestre $1982(28)$ :

- emprunt $1982-92$ à $7 \%$ de 100 millions ća francs;

- placement privé $1982-87$ à $8 \%$ de 50 millions de francs.

Au cours de la réunion annuelle de la BID (29 au 31 mars 1982 à Carthagène/ Colombie), la sixième augmentation du capital fut au centre des délibérations. Alors que les $\mathrm{EU}$ se prononçaient pour une politique financière restrictive de cette banque de développement, les pays membres de la région insistaient pour obtenir une extension des crédits. Des divergences d'opinion se manifestèrent également quant à la part du capital à fournir par les pays membres et concernant la politique de promotion, c'est-à-dire l'accès des divers pays aux crédits de la banque. Les discussions se poursuivent.

La Suisse se prononce pour une substantielle augmentation du capital en faveur de l'autodiscipline des plus riches pays membres de la région. Si l'on réduisait la part du capital à fournir, la situation de la banque sur les marchés des capitaux pourrait en souffrir. La Suisse plaide aussi pour obtenir une meilleure représentation des membres non régionaux à la direction de la banque. Elle demanda qu'un troisième siège à la direction soit attribué à ces pays. La Suisse a versé l'année passée au fonds spécial de la banque une contribution de 7,3 millions de francs (29).

La banque asiatique de développement (ADB), dont la Suisse fait partie depuis 1967, a contracté en Suisse au cours de 1981 et du premier semestre 1982 les emprunts suivants :

- emprunt $1981-91$ à $7,75 \%$ de 80 millions de francs;

- emprunt 1981-90 à $8 \%$ de 80 millions de francs, d'un consortium bancaire suisse sous la direction du Crédit suisse;

- emprunt 1982-92 à 7\% de 100 millions de francs. 
Fin avril, lors de la réunion annuelle de I'ADB à Manille, on a décidé de refinancer pour la troisième fois le Fonds asiatique de développement (ADF) au moyen duquel la banque ouvre aux pays les plus pauvres des crédits à des conditions très favorables. Ce refinancement mobilise 3,2 milliards de dollars EU. La Suisse fournit 10 millions de francs. En termes réels, I'offre de crédits reste ainsi à son niveau antérieur. A l'origine, la banque espérait pouvoir augmenter ses crédits, proposition repoussée après un an de négociations à cause de l'attitude restrictive des Etats-Unis dont la part proportionnelle au financement de l'institution est maintenant plus faible qu'avant (30).

Lors de la réunion annuelle de la Banque africaine de développement (BAD), du 5 au 8 mai 1982 à Lusaka/Zambie, décision a été prise d'accueillir des membres non régionaux. La Suisse avait déjà décidé en 1980 de faire partie de cette banque de développement. Le troisième refinancement du Fonds africain de développement (FAD) s'est opéré avec succès. Des apports de 1,07 milliard de dollars EU permettent au fonds de poursuivre son activité, mais sans réelle progression des moyens disponibles.

La contribution de la Suisse s'élève à 26,9 millions de francs. La Suisse peut en outre désigner pour les deux années à venir un directeur exécutif de cette banque de développement (31).

\section{COOPERATION TECHNIQUE ET AIDE FINANCIERE}

Financée à partir du crédit-cadre actuel, l'aide technique fournie en 1981 de manière bilatérale s'est concentrée sur l'agriculture et les pays où les engagements sont massifs. L'aide financière multilatérale est allée en grande partie au PNUD, au FAD et à I'IFAD.

En 1981, la DDA a travaillé sur la toile de fond constituée par le crédit-cadre de 1.650 millions de francs accordé en 1980. La DDA est l'organe de la Confédération qui canalise à peu près toute la coopération technique et toute I'aide financière fournie par des fonds publics. A fin juin 1982, le crédit de 1980 avait été sollicité pour un montant de 549 millions de francs en faveur de projets dont certains se réaliseront jusqu'en 1986 (32).

Disposant de 415 collaborateurs à la centrale et à l'étranger, la DDA a réalisé l'an passé 435 projets bilatéraux, dont 50 étaient des projets nouveaux. 406 projets sont du ressort de la coopération technique et 29 sont d'aide financière (33).

Le tableau suivant reproduit les engagements de la DDA en 1981 au double titre de la coopération technique et de l'aide financière : 


\begin{tabular}{lccc} 
& $\begin{array}{l}\text { Coopération } \\
\text { technique }\end{array}$ & $\begin{array}{l}\text { Aide } \\
\text { financière }\end{array}$ & Total \\
\hline Activités bilatérales & $\begin{array}{r}153,5 \\
37,2\end{array}$ & $\begin{array}{r}46,5 \\
54,0\end{array}$ & $\begin{array}{r}200,0 \\
91,2\end{array}$ \\
\cline { 2 - 4 } Activités multilatérales & 190,7 & 100,5 & 291,2 \\
\hline
\end{tabular}

Source : Rapport de gestion du Conseil fédéral 1981, p. 30.

\section{Coopération bilatérale au développement}

La coopération bilatérale au développement (200 millions) et la coopération technique (153,5 millions) sont depuis plusieurs années les principales formes d'expression de la coopération suisse au développement. En 1981, la DDA a réalisé 188 projets en son nom propre; 61 initiatives de coopération technique ont été données en régie à des institutions ou à des firmes suisses; 102 projets d'œuvres d'entraide privées, de corporations ou d'institutions publiques ont bénéficié d'une contribution financière. Des versements ont encore été effectués en faveur de 84 projets d'aide technique ou financière présentés par des organisations internationales. La collaboration avec l'économie privée s'exprime surtout sous forme d'achats de matériel et de commandes passées à des bureaux d'ingénierie ou à des firmes d'ingénieurs-conseils (27 millions de francs en 1982) (34).

La répartition par continents de la coopération technique bilatérale et de I'aide financière s'est légèrement modifiée en 1981, I'Afrique absorbant, aux dépens de I'Asie, une part des versements de 5,5\% plus élevée que I'année précédente. Les versements en faveur de l'Asie ont également diminué de volume (voir les statistiques).

\section{PROJETS DE COOPERATION TECHNIQUE BILATERALE ET D'AIDE FINANCIERE EN 1981 : REPARTITION PAR CONTINENTS \\ (versements nets en millions de francs)}

\begin{tabular}{lcr} 
Région & $\begin{array}{l}\text { Millions } \\
\text { de francs }\end{array}$ & $\%$ \\
\hline Afrique & 90,4 & 45,2 \\
Amérique latine & 28,2 & 14,1 \\
Asie & 57,7 & 28,9 \\
Europe & 1,8 & 0,9 \\
Non classés & 21,8 & 10,9 \\
\cline { 2 - 3 } TOTAL & 200,0 & 100,0 \\
\hline
\end{tabular}

Source : Rapport annuel de la DDA, 1981. 
Les dix-sept pays d' "engagements massifs" de la coopération suisse au développement ont absorbé en 1981 82,3\% des versements effectués. Leur répartition par groupes de revenus se présente ainsi :

Coopération technique bilatérale et aide financière :

\section{VERSEMENTS CLASSES SELON LE REVENU DES PAYS BENEFICIAIRES}

(en millions de francs)

\begin{tabular}{|c|c|c|c|c|}
\hline Groupes de pays & $\begin{array}{l}\text { Coopération } \\
\text { technique }\end{array}$ & $\begin{array}{l}\text { Aide } \\
\text { financière }\end{array}$ & TOTAL & $\%$ \\
\hline Pays les moins avancés & 54,9 & 31,1 & 86,0 & 43 \\
\hline \multicolumn{5}{|l|}{ Autres pays à faible revenu } \\
\hline (PNBde moins de 500 \$ par tête) & 33,6 & 7,8 & 41,4 & 20,7 \\
\hline \multicolumn{5}{|l|}{ Pays à revenu moyen } \\
\hline (PNB de plus de 500 \$ par tête) & 22,9 & 2,4 & 25,3 & 12,6 \\
\hline Projets régionaux et non classés & 42,1 & 5,2 & 47,3 & 23,7 \\
\hline TOTAL & 153,5 & 46,5 & 200,0 & 100,0 \\
\hline
\end{tabular}

Source : Rapport annuel de la DDA 1981, tableau 6. Les regroupements par pays adoptés ici correspondent aux définitions de l'OCDE et sont basés sur le PNB de 1979.

A relever dans la répartition par secteurs d'activité, l'augementation enregistrée par l'agriculture dont la part passe à $35 \%$. Peu de changements dans les autres secteurs. Par contre, les projets pluridisciplinaires prennent une place moins importante (voir les statistiques).

\section{Aide associée et cofinancement}

L'aide associée et le cofinancement sont d'autres aspects de la coopération bilatérale au développement. Par aide associée, il faut entendre : "Financement d'un projet qui est réalisé et géré par une organisation internationale, mais choisi et contrôlé par la DDA" (35). Le cofinancement signifie que le projet est financé par plusieurs donateurs (intervenant au plan bilatéral et/ou multilatéral) et domiciliés hors du pays bénéficiaire; I'opération se déroule sur la foi d'un contrat signé avec l'agrément du bénéficiaire.

La Suisse fournit une aide associée en collaboration avec plusieurs organisations spécialisées de l'ONU et avec des institutions comme la FAO et le BIT. A la fin 1981, cinquante-cinq projets de ce genre étaient en cours de réalisation, dont vingt-trois sous la direction de la FAO, dix sous la direction 
du BIT et huit assumés par I'UNICEF. Les versements effectués en 1981 au compte de l'aide associée et du cofinancement ont été de 42,6 millions de francs, comme indiqué sur le tableau ci-dessous. Les principaux bénéficiaires en ont été des institutions de I'ONU (FAO, BIT, UNICEF), des instituts internationaux de recherches et des institutions de financement du développement (banques régionales de développement et la filiale de la Banque mondiale AID).

La DDA assume une responsabilité partielle dans le financement de quelques projets des banques régionales de développement et de I'AID. II s'agit de projets que la DDA choisit elle-même. Le financement est en principe non lié. Le projet est réalisé par le pays bénéficiaire sous surveillance de la banque de développement, les entreprises suisses pouvant se manifester lors de la mise au concours du projet.

\section{AIDE ASSOCIEE ET COFINANCEMENT AVEC DES ORGANISATIONS INTERNATIONALES}

(Versements nets en millions de francs, 1981)

\begin{tabular}{|c|c|c|c|}
\hline & $\begin{array}{l}\text { Aide } \\
\text { associée }\end{array}$ & $\begin{array}{l}\text { Cofinan- } \\
\text { cement }\end{array}$ & TOTAL \\
\hline Institutions des Nations Unies, ONU & 18,0 & - & 18,0 \\
\hline $\begin{array}{l}\text { Organisation de I'ONU pour l'alimentation } \\
\text { et l'agriculture (FAO) }\end{array}$ & 6,6 & - & 6,6 \\
\hline $\begin{array}{l}\text { Oeuvre de secours aux enfants de l'ONU } \\
\text { (UNICEF) }\end{array}$ & 4,0 & - & 4,0 \\
\hline Bureau international du travail (BIT) & 3,3 & - & 3,3 \\
\hline $\begin{array}{l}\text { Conférence des Nations Unies pour le } \\
\text { commerce et le développement } \\
\text { (CNUCED) }\end{array}$ & 0,8 & - & 0,8 \\
\hline Autres & 3,3 & - & 3,3 \\
\hline Institutions de financement du développement & - & 8,6 & 8,6 \\
\hline Agence internationale du développement (AID) & - & 5,0 & 5,0 \\
\hline Banque africaine de développement (BAD) & - & 0,5 & 0,5 \\
\hline Banque asiatique de développement ( $A D B$ ) & - & 3,1 & 3,1 \\
\hline Instituts internationaux de recherches & 14,9 & - & 14,9 \\
\hline $\begin{array}{l}\text { Groupe consultatif pour la recherche } \\
\text { agricole internationale (GCRAI) }\end{array}$ & 12,6 & - & 12,6 \\
\hline Autres & 2,3 & - & 2,3 \\
\hline Autres organisations internationales & 1,1 & - & 1,1 \\
\hline TOTAL & 34,0 & 8,6 & 42,6 \\
\hline
\end{tabular}

Source : Rapport de gestion du Conseil fédéral 1981, p. 39. 
En 1980, les banques interaméricaine et asiatique de développement avaient reçu des contributions non remboursables pour des projets d'aide technique et de petits projets d'aide financière; en août 1981, la banque asiatique de développement a bénéficié d'une aide de même nature (36).

\section{Coopération multilatérale au développement}

La coopération technique multilatérale a absorbé 37,2 millions de francs en 1981 et l'aide financière 54 millions de francs, soit un total de 91,2 millions de francs pour les deux postes. Le PNUD en a reçu la plus grande part avec 30,2 millions de francs. Viennent ensuite le Fonds africain du développement (26,9 millions) et son pendant asiatique (10 millions).

En 1981, le PNUD n'a disposé que d'environ 550 millions de dollars, la Suisse couvrant $2,1 \%$ des versements (37). La DDA coordonne et administre les crédits de l'aide financière multilatérale fournis à la fois par elle-même et I'OFAEE. A part les contributions versées aux différents fonds régionaux de développement, il convient de relever le refinancement du Fonds international de développement agricole (FIDA). Les négociations furent longues; il s'agissait de fixer la proportion des prestations dues par les pays de I'OPEP et ceux de l'OCDE; la Suisse demanda que l'activité du fonds se poursuive au moins au niveau antérieur; elle est représentée depuis 1981 au conseil d'administration du FIDA par un adjoint au directeur. Les pays de I'OCDE, de I'OPEP et les autres pays en développement disposent du même nombre de voix au FIDA qui a pour but de développer les productions vivrières indigènes et de travailler à la satisfaction des besoins essentiels (38).

A fin juin 1982, le Conseil fédéral a autorisé le versement d'une contribution suisse de 28,44 millions de francs ( 15,5 millions de dollars) au premier refinancement du FIDA, ce qui représente $2,5 \%$ de l'apport total des pays de I'OCDE. Lors de sa 5ème session (du 19 au 22 janvier 1982 à Rome), le conseil des gouverneurs du FIDA a choisi Rome comme siège définitif de I'institution. Devisé à 1.100 millions de dollars, le refinancement du fonds est pour $58 \%$ à la charge des pays de l'OCDE et pour $42 \%$ à la charge des pays de I'OPEP. II s'effectue présentement sans participation des EtatsUnis qui en ont bien admis le principe, mais sans avoir porté à leur budget les montants nécessaires (39). 
Coopération technique multilatérale et aide financière

CONTRIBUTIONS A DES ORGANISATIONS INTERNATIONALES

(Versements nets en millions de francs, 1981)

\begin{tabular}{|c|c|c|c|}
\hline & $\begin{array}{l}\text { Coopération } \\
\text { technique }\end{array}$ & $\begin{array}{l}\text { Aide } \\
\text { financière }\end{array}$ & TOTAL \\
\hline Organisation des Nations Unies (ONU) & 37,2 & 6,8 & 44,0 \\
\hline $\begin{array}{l}\text { Programme de développement } \\
\text { de I'ONU (PNUD) }\end{array}$ & 30,2 & - & 30,2 \\
\hline Fonds pour les pays les moins avancés & 4,0 & $\cdot$ & 4,0 \\
\hline Fonds pour la science et la technologie & 0,8 & - & 0,8 \\
\hline Fonds d'équipement de l'ONU (FCDNU) & $\cdot$ & 3,8 & 3,8 \\
\hline Fonds d'aide aux populations ONU (FAPNU) & - & 3,0 & 3,0 \\
\hline $\begin{array}{l}\text { Fonds spécial de l'Organisation mondiale } \\
\text { de la santé pour l'étude des maladies } \\
\text { tropicales (OMS) }\end{array}$ & 1,7 & - & 1,7 \\
\hline Autres fonds spéciaux de I'ONU & 0,5 & - & 1,7 \\
\hline $\begin{array}{l}\text { Banques régionales de développement } \\
\text { et leurs fonds spéciaux }\end{array}$ & $\cdot$ & 47,2 & 47,2 \\
\hline Fonds africain de développement (FAD) & - & 26,9 & 26,9 \\
\hline Banque asiatique de développement (ADB) & - & 2,3 & 2,3 \\
\hline Fonds asiatique de développement (ADF) & - & 10,0 & 10,0 \\
\hline Banque interaméricaine de développement (BID) & $\cdot$ & 0,7 & 0,7 \\
\hline Fonds pour opérations spéciales de la BID & - & 7,3 & 7,3 \\
\hline TOTAL & 37,2 & 54,0 & 91,2 \\
\hline
\end{tabular}

Source : Rapport annuel de la DDA 1981, tableau 16.

\section{AIDE HUMANITAIRE}

Au cours de leur session d'hiver 1981, le 3 décembre, les Chambres fédérales ont accordé un nouveau crédit-cadre de 360 millions de francs pour l'aide humanitaire internationale, sur la foi du message publié par le Conseil fédéral. Ce nouveau crédit-cadre était devenu indispensable du fait que le précédent, d'un montant de $\mathbf{2 7 0}$ millions de francs, arrivait à échéance à fin mars 1982. Le nouveau crédit-cadre a une durée minimum de trois ans. II est prévu d'en ventiler ainsi le montant entre les différents secteurs de l'aide humanitaire (40) : 
Versements à des organisations internationales et à des œuvres d'entraide de portée internationale

Corps suisse d'aide en cas de catastrophes

Aide alimentaire à base de produits laitiers suisses

Aide en céréales

Réserve pour cataclysmes extraordinaires, y compris obligations envers les secours d'urgence de l'aide alimentaire internationale

En décembre 1981, le Parlement a en outre accepté de porter à 20 millions de francs la contribution annuelle de la Suisse au CICR (41).

Les versements effectués en 1981 au titre de l'aide humanitaire s'établissent ainsi :

\section{AIDE HUMANITAIRE 1981}

(en millions de francs)

Engagements du Corps des volontaires pour secours en cas de catastrophes à l'étranger

Versements ordinaires et contributions extraordinaires à des organisations internationales et des œuvres d'entraide de portée internationale (y compris renouvellement du matériel de secours)

Aide alimentaire

Produits laitiers et céréales suisses

(y compris versements en espèces pour

I'achat et le transport des aliments)

TOTAL

92,15

Source : Rapport annuel de la DDA 1981, tableau 17. Les montants relevés comprennent 2,5 millions de francs finançant des initiatives qui n'ont pas été entreprises dans des pays en développement, comme la Pologne, par exemple. 


\section{Aide alimentaire internationale}

La Suisse a fourni une contribution de 46,2 millions de francs à l'aide alimentaire internationale en 1981. Ce montant représente la moitié des sommes engagées l'an passé dans les opérations d'aide humanitaire en général. A part les dons en espèces, 3.140 tonnes de produits laitiers suisses, 21.151 tonnes de produits à base de céréales ainsi que d'autres aliments en petites quantités ont été cédés gratuitement. Leur distribution s'est effectuée par des vaies bilatérales et multilatérales. La moitié environ des envois est allée aux secours d'urgence et à l'aide aux réfugiés (42).

Une étude de l'Institut d'éthique sociale de la Fédération des Eglises protestantes de Suisse et du Service d'information tiers monde i3m estime que I'aide alimentaire est certes nécessaire, mais qu'elle n'en reste pas moins problématique (43). Les auteurs sont d'avis qu'une aide humanitaire rapide ne devrait être accordée que pour les secours d'urgence en cas de catastrophes. L'évaluation des dons de lait en poudre reste insuffisante dans un domaine où se mêlent les intérêts de l'économie suisse et les besoins des territoires en proie à la famine. Cet affrontement est encore plus accusé à l'occasion des livraisons de céréales suisses. Le programme d'essai à base de conserves suisses de poisson blanc se heurte à beaucoup d'incompréhension (44).

\section{Aide humanitaire opérationnelle}

Le Corps des volontaires pour l'aide en cas de catastrophes à l'étranger est le bras opérationnel de l'aide humanitaire. Il a été peu engagé en 1981, vu l'absence de cataclysmes. Des interventions ont eu lieu au bénéfice des réfugiés de Thaïlande, des Philippines, du Pakistan, du Cameroun et de la Somalie.

Après plusieurs années de travail, les chantiers de reconstruction ouverts au Guatémala après le tremblement de terre de février 1976 ont pu être fermés, tout comme ceux d'El Asnam (Algérie). Diverses interventions en vue d'améliorer I'infrastructure ont eu lieu au Ghana, au Zimbabwe et en Guinée équatoriale.

Le total des dépenses au titre de l'aide aux réfugiés s'est élevé en 1981 à 22,65 millions de francs, dont 12,79 millions ont été acheminés par l'aide multilatérale. Au compte de l'aide bilatérale, on relève 5,84 millions de francs dépensés en Afrique (Somalie) et 3,68 millions de francs utilisés en Asie (Pakistan et Thaïlande) (45). En juin 1982, le Conseil fédéral a accordé un crédit supplémentaire de 5 millions de francs pour secours d'urgence à la population civile du Liban victime de la guerre (46).

A fin 1981, Arthur Bill, le premier délégué du Conseil fédéral à l'aide en cas de catastrophes à l'étranger, a pris sa retraite. Le Conseil fédéral a désigné son successeur en la personne d'Eduard Blaser (47). 


\section{BOURSES}

Le crédit-cadre de 9,2 millions de francs du 19 décembre 1980 donne à I'Office fédéral des affaires culturelles du DFI de quoi accorder des bourses aux étrangers poursuivant leurs études en Suisse. La plupart des bénéficiaires proviennent des pays en développement. 3,3 millions de francs ont été dépensés dans ce domaine en 1981, ce montant englobant un versement de 920.000 francs prélevé par la DDA sur son crédit pour les bourses. 3,8 millions de francs sont prévus pour 1982, y compris l'apport de la DDA. Le DFI a accordé environ deux cents bourses en 1981 (48).

En vertu de la coopération technique bilatérale et de l'aide associée, deux cent quatre-vingt-deux boursiers venant des pays en développement ont poursuivi leur formation en Suisse au cours de 1981. La DDA a engagé à cet effet 2,7 millions de francs. Les bourses de la DDA sont accordées à des personnes jouissant déjà d'une expérience professionnelle, pour des cours de perfectionnement.

En 1981, la plupart des boursiers ont reçu leur formation dans le secteur des services (hôtellerie, postes et télécommunications, santé publique) ou dans le domaine de l'industrie et de la technique. La majorité des boursiers (67\%) venait de pays africains et $20 \%$ de pays asiatiques. Les femmes étaient représentées à raison de $11 \%$ du total. Au cours de 1980-81, une enquête a été menée pour connaître la situation professionnelle actuelle des anciens boursiers de la DDA. Ils travaillent en grande majorité dans leur pays d'origine et dans les domaines où ils ont été formés en Suisse (49).

\section{PRESTATIONS DES CANTONS ET DES COMMUNES}

En 1981, la contribution des cantons et des communes au financement de la coopération publique au développement s'est élevée à 6,1 millions de francs (ou 1,35\%); ces prestations sont allées à des projets présentés par des organisations privées. Le total des montants fournis par les cantons a été de 2,78 millions de francs. Les apports les plus importants sont venus des cantons de Genève ( 898.000 francs), Bâle-Ville (507.000 francs) et Zurich ( 255.000 francs). Diverses communes ont fourni en tout 3,34 millions de francs. Sept d'entre elles ont donné plus de 100.000 francs : Genève, Zurich, Berne, Küsnacht $(\mathrm{ZH})$, Zollikon $(\mathrm{ZH})$, Lancy $(\mathrm{GE})$ et Kloten $(\mathrm{ZH})$ (50).

Divers cantons (Bâle-Ville, Jura, Vaud) et une commune (Münsingen/BE) financent leurs propres projets dans des pays en développement. Leurs 
prestations, y compris le financement complémentaire qu'ils reçoivent de la Confédération, s'est élevé en 1981 à 0,88 million de francs (51).

En 1980 a été lancée à Genève une initiative dite "Initiative 0,7" qui demande la création d'un fonds cantonal d'aide au développement financé au moyen d'un prélèvement de $0,7 \%$ du revenu cantonal. La proportion de $0,7 \%$ serait atteinte progressivement au cours des dix prochaines années.

Le Grand conseil s'est prononcé le 21 janvier 1982 en faveur de l'initiative et la votation aura lieu le 26 septembre. Les ressources du fonds financeront des projets de coopération technique allant dans le sens des efforts entrepris par les pays en développement eux-mêmes et profitant aux régions et aux groupes humains les plus défavorisés. Les projets devisés à plus de 250.000 francs devront être acceptés par le Grand conseil et soumis au référendum. Si la proportion des chômeurs enregistrés dans le canton ou en Suisse atteint $0,5 \%$ ou si une région de la Suisse connaît des difficultés économiques particulières, I'aide prévue pourra être ramenée à $0,6 \%$ du revenu cantonal (52).

Une motion présentée au parlement jurassien a demandé que $0,1 \%$ du budget courant soit consacré à la coopération au développement et cela aussi longtemps que la Suisse ne donnerait pas $0,7 \%$ de son PNB pour la coopération publique au développement (53).

\section{LA COOPERATION DES ORGANISATIONS PRIVEES AU DEVELOPPEMENT}

La coopération des milieux privés au développement ne peut être rangée parmi les prestations de la Confédération. Elle y est tout de même associée à maints égards. Les œuvres d'entraide privées reçoivent de la Confédération de quoi compléter le financement de leurs projets ou des projets à réaliser en régie. Dans la discussion politique sur le développement, les représentants des œuvres d'entraide privées sont des interlocuteurs de poids.

Les prestations des œuvres d'entraide privées et des organisations travaillant au développement ne se bornent pas aux travaux de coopération technique et d'aide humanitaire. Ces organisations se manifestent aussi en Suisse. Diverses organisations ne réalisent même aucun projet dans les pays en développement, leur mission étant d'informer la population de tout ce qui a trait au développement.

Au cours de 1981, les œuvres d'entraide privées, à vocation religieuse ou d'attitude neutre, sont parvenues à réunir des dons d'un montant total de 105,6 millions de francs pour financer leurs activités. Si le montant de l'aide publique au développement place la Suisse au-dessous de la moyenne enregistrée dans les pays de l'OCDE, les dons recueillis par les œuvres d'entraide la mettent au premier rang avec la Norvège. Dans ces deux pays, les presta- 
tions des œuvres d'entraide privées représentaient en 1981 0,06\% du PNB (54).

La fondation d'une "nouvelle organisation" a été envisagée en 1981 dans le but de maîtriser le volume croissant des travaux de coopération au développement et de maintenir la qualité et le nombre des projets bilatéraux malgré le blocage du personnel décrété par la Confédération. Cet organisme devint rapidement l'objet d'une controverse soutenue et nourrie en particulier par les arguments des parlementaires bourgeois et des bureaux d'ingénierie.

La commission consultative de la coopération internationale au développement et la commission des affaires étrangères du Conseil des Etats et du Conseil national s'étant occupées de ce projet, le Conseil fédéral se prononça le 21 décembre 1981 pour la conclusion d'un accord de coopération entre la Confédération et "Intercoopération". Cette association fut créée au début de 1982 par sept œuvres d'entraide (Caritas, Helvetas, I'EPER, I'OSEO, Swissaid, Swisscontact et Croix-Rouge). La Confédération avait finalement renoncé à en faire partie pour des raisons juridiques. Un accord détermine les termes d'une étroite collaboration entre la Confédération et "Intercoopération". La documentation fournie à la presse à cette occasion précise : "La Confédération et les œuvres d'entraide fournissent à l'association un prêt de 500.000 francs de part et d'autre (1 million de francs au total); la Confédération fournit en outre un montant non remboursable pour aider au démarrage de l'association. Le volume des projets transférés à l'association par la Confédération se situera au cours des prochaines années dans un ordre de grandeur d'environ 13 à 15 millions de francs. Au début, dix à douze personnes travailleront au siège de l'association, un nombre plus élevé de collaborateurs devant être engagés sur le terrain, dans les projets à réaliser dans les pays en développement."

"L'activité de l'association portera surtout sur des projets agricoles (programmes d'élevage et de développement de la production fourragère), sylvicoles (reboisements aux abords des villages et lutte contre l'érosion) et de développement rural à la base dans différents domaines. Ces projets devront dans toute la mesure du possible tenir compte des impératifs techniques et être réalisés en étroite collaboration avec les populations rurales et les organisations locales. Normalement, ils ne doivent pas être onéreux, mais conçus de manière à mobiliser beaucoup de main-d'œuvre. Ils seront préparés et contrôlés avec soin" (55). 